\title{
Effect of Fungicides and Herbicides against Rhizoctonia solani f. sp. sasakii Exner Causing Banded Leaf and Sheath Blight in maize (Zea mays L.)
}

\author{
M. Madhavi ${ }^{*}$, P. Narayan Reddy ${ }^{2}$, K. Manohar ${ }^{3}$ and Ch. Aruna Kumari ${ }^{4}$
}

${ }^{1}$ Dept. of Plant Pathology, Agricultural College, Polasa, Jagtial (Dist), Telangana, (505 529), India

${ }^{2,3}$ Dept. of Plant Pathology, College of Agriculture, Rajendranagar, Hyderabad, Telangana (500 030), India

${ }^{4}$ Dept. of Crop Physiology, Agricultural College, Polasa, Jagtial (Dist) Telangana (505 529), India

\section{Corresponding Author}

M. Madhavi

e-mail: madhagonii@gmail.com
Article History

Article ID: 3 C0531

Received in $19^{\text {th }}$ October, 2017

Received in revised form $21^{\text {st }}$ December, 2017

Accepted in final form $27^{\text {th }}$ January, 2018

\begin{abstract}
Nine fungicides, one antibiotic and four herbicides including both systemic and non systemic were screened against Rhizoctonia solani f.sp. sasakii Exner (Thanatephorus sasakii (Shirai) Tu and Kimbrough causing Banded Leaf and Sheath Blight (BLSB) in maize. All the 27 isolates of $R$. solani from maize and one from rice were found to be highly sensitive to systemic fungicides propiconazole, hexaconazole, tebuconazole and carbendazim which showed cent \% mycelial inhibition followed by new molecules i.e. cabriotop, metiram and pyraclostrobin at recommended and half the recommended concentrations. The isolate RS 28 from rice showed 78.88 and $90.00 ; 76.16$ and $83.33 \%$ inhibition with respect to cabriotop and metiram at recommended and half the recommended concentrations, respectively. Among the non systemic fungicides mancozeb was relatively inhibitorier than thiram. Mancozeb at recommended concentration showed $72.22 \%$ inhibition of mycelial growth in virulent isolates RS11 and RS12 from Khammam district. At recommended concentration validamycin, completely inhibited the radial growth of the isolates RS 4, RS 22, RS 24 and RS 27 while $80.00 \%$ inhibition in rice isolate. Among the herbicides tested, systemic herbicide pendimethalin was highly effective with per cent inhibition in all the isolates except for the isolate RS12 from Yellandu mandal of Khammam district at half the recommended concentration. While the remaining herbicides i.e. atazine, 2,4-D and paraquat exhibited differential reaction indicating variability among the isolates at both the concentrations tested. The rice isolate reacted in a similar way as that of maize isolates against different fungicides and herbicides tested.
\end{abstract}

Keywords: Maize, Rhizoctonia solani, BLSB, sensitivity, fungicides, antibiotic, herbicides

\section{Introduction}

Maize (Zea mays L.) the third most important cereal crops in the world's agricultural economy had highest genetic yield potential and is commonly called as queen of cereals. One of the main deterrents to high grain yield in maize is its susceptibility to several diseases. The banded leaf and sheath blight (BLSB) caused by Rhizoctonia solanif. sp. sasakii Exner, (Tel: Thanatephorus sasakii (Shirai) Tu and Kimbro) is a very destructive disease of maize and is gaining economic importance especially in several hot and humid tropical maize growing areas worldwide (Akhtar et al., 2009; Subash Subedi, 2015). The disease has become a major constraint in breeding programme because of the non-availability of widely adapted and stable source of resistance to BLSB. Therefore, in the absence of suitable resistant donor, chemical control which mostly relies on the use of fungicides is the only alternative to check the disease and to prevent economic losses. Knowledge of the effectiveness of particular compounds is important for achieving effective disease control. Since the seriousness of disease warrants chemical protection, it is important to explore chemical molecule to avoid build up of resistance in the pathogen. Several systemic as well as non systemic fungicides have been used which posses good control against Rhizoctonia solani.

Neverthless, several cultivated and wild hosts are very important sources of survival of the R.solani pathogen involving the role of weeds in the recurrence of the disease. If, herbicides applied to manage the weed problems in the crop, are toxic to a potential pathogen, or if they place the pathogen at a competitive disadvantage, their capacity to induce the disease may be reduced (Altman and Campbell, 1977). Investigations and reviews have been done from time to time to know the effects of a herbicidal compound on a fungal pathogen (Black et al., 1996; Zidan et al., 1998). Such studies have gained importance in the era of sustainable agriculture involving minimum use of chemical pesticides coupled with increased pressure on plant pathogens and pests. 
In recent years, the disease occurrence was wide spread in almost all major maize growing areas of Telangana and Andhra Pradesh states with different intensities in most of the popular cultivars. Twenty seven samples of maize exhibiting BLSB symptoms were collected from nine major maize growing districts of Telangana and Andhra Pradesh states at the rate of three different mandals in each district. The pathogen $R$. solani isolates were isolated, identified and designated as $\mathrm{RS} 1$ to RS27. One isolate from rice collected from RangaReddy district was designated as RS28 (Table 1). In the present study nine fungicides, one antibiotic (Validamycin) and four herbicides, including three systemic (pendimethalin, atrazine and 2, 4-D) and one contact (paraquat) at recommended and half the recommended concentrations were evaluated under in vitro condition for their bio-efficacy against the $R$. solani isolates to select the effective fungicide and herbicide for disease control in addition to the weed control.

\section{Materials and Methods}

The fungicides and herbicides used in the present study along with particulars of trade name, common name, chemical name and active ingredient of the chemical in the formulation and source of supply are presented in (Table 2). Poisoned food technique (Nene and Thapliyal, 1993) was adopted to determine the sensitivity of the 28 isolates of $R$.solani.

$\%$ growth inhibition was calculated in each treatment by comparison with control plates (Bliss, 1934).

$\mathrm{I}=[(\mathrm{C}-\mathrm{T}) / \mathrm{C}] \times 100$

where I=Percent inhibition

$\mathrm{C}=$ Colony diameter in control $(\mathrm{mm})$

$\mathrm{T}=$ Colony diameter in treatment $(\mathrm{mm})$

\section{Results and Discussion}

3.1. Bio-assay of $R$. solani isolates with Fungicides and Antibiotic

Out of the nine fungicides, seven fungicides were systemic and remaining were non systemic. Among the seven systemic fungicides, four fungicides viz carbendazim, propiconazole, tebuconazole and hexaconazole were highly effective and completely inhibited the radial growth of all the isolates including rice isolate at both recommended and half the recommended concentrations. While the new molecules pyraclostrobin and cabriotop were relatively less effective and showed differential reaction indicating variability among the isolates at both the concentrations tested.

Most of the R.solani isolates reacted very sensitively with the systemic fungicide cabriotop, wherein the isolates RS3 (Gajwal mandal of Medak district), RS4, RS5, RS6 (Nizamabad), RS7, RS8 (Karimnagar), RS13, RS14, RS15 (Warangal), RS19, RS20, RS21 (Guntur) and RS27 (Thatipadu mandal of Kurnool district) showed complete inhibition at recommended and half the recommended concentrations. The virulent isolate
Table 1:. Details of Rhizoctonia solani f sp sasakii isolates collected from major maize growing districts of Telangana and Andhra Pradesh States

\begin{tabular}{|c|c|c|c|c|}
\hline $\begin{array}{l}\text { SI. } \\
\text { No. }\end{array}$ & $\begin{array}{l}\text { I so - } \\
\text { lates }\end{array}$ & $\begin{array}{l}\text { Place of col- } \\
\text { lection }\end{array}$ & District & Variety/Cultivar \\
\hline 1. & RS1 & Pragnyapur & Medak & Kaveri-225 \\
\hline 2. & RS2 & Doulatabad & Medak & Pioneer-30V92 \\
\hline 3. & RS3 & Gajwel & Medak & Kanchana \\
\hline 4. & RS4 & Armur & Nizamabad & C-Tex \\
\hline 5. & RS5 & Kamareddy & Nizamabad & Kaveri \\
\hline 6. & RS6 & Mortad & Nizamabad & Kanchana \\
\hline 7. & RS7 & Jagityal & Karimnagar & C-Tex/Prince \\
\hline 8. & RS8 & Metpally & Karimnagar & Pioneer \\
\hline 9. & RS9 & Raichal & Karimnagar & Kaveri Gold \\
\hline 10. & RS10 & Bonakal & Khammam & Kanchana \\
\hline 11. & RS11 & Chintakani & Khammam & Pioneer \\
\hline 12. & $\mathrm{RS} 12$ & Yellandu & Khammam & Kaveri /C-Tex \\
\hline 13. & RS13 & Janagoan & Warangal & Yecca \\
\hline 14. & RS14 & Atmakur & Warangal & Kanchana \\
\hline 15. & RS15 & Hasanparthy & Warangal & Pioneer \\
\hline 16. & RS16 & Vatsavai & Krishna & Kaveri-50 \\
\hline 17. & RS17 & Tiruvur & Krishna & Yecca \\
\hline 18. & RS18 & Nuziveedu & Krishna & Pioneer-30V 92 \\
\hline 19. & RS19 & Tenali & Guntur & Pioneer/Kargil \\
\hline 20. & RS 20 & Mangalgiri & Guntur & Kaveri 255 \\
\hline 21. & RS 21 & Kolipara & Guntur & Yecca \\
\hline 22. & RS22 & Eluru & $\begin{array}{l}\text { West Go- } \\
\text { davari }\end{array}$ & Pioneer-30V 92 \\
\hline 23. & RS23 & $\begin{array}{l}\text { Jangared- } \\
\text { dygudem }\end{array}$ & $\begin{array}{l}\text { West Go- } \\
\text { davari }\end{array}$ & Kanchana \\
\hline 24. & RS24 & Jeelugumilli & $\begin{array}{l}\text { West Go- } \\
\text { davari }\end{array}$ & Kaveri-255 \\
\hline 25. & RS25 & Nandikotkur & Kurnool & Kaveri Gold \\
\hline 26. & $\mathrm{RS} 26$ & Atmakur & Kurnool & Kargil \\
\hline 27. & RS27 & Thatipadu & Kurnool & Pioneer \\
\hline 28. & $\begin{array}{l}\text { R S } 28 \\
\text { (Rice) }\end{array}$ & $\begin{array}{l}\mathrm{R} \text { a j e } \mathrm{n}- \\
\text { dranagar }\end{array}$ & $\begin{array}{l}\text { RangaRed- } \\
\text { dy }\end{array}$ & ВРТ-5204 \\
\hline
\end{tabular}

RS11 from Chintakani mandal of Khammam district recorded least inhibition of 61.11 and 51.41 at recommended and half recommended concentration, respectively. Rice isolate RS28 recorded $90.00 \%$ inhibition at recommended concentration and was on par with maize isolates RS16 and RS18 from Krishna district (Table 3).

All the isolates showed differential sensitivity against 


\begin{tabular}{|c|c|c|c|c|c|}
\hline & $\begin{array}{l}\text { T r a d e } \\
\text { Name }\end{array}$ & Common Name & Chemical Name & $\begin{array}{l}\text { Active In- } \\
\text { gredients }\end{array}$ & Source of supply \\
\hline 1. & Tilt & Propiconazole & $\begin{array}{l}\text { (=)- 1-[ 2- (2,4 - dichlorophenyl)- } 4 \text { propyl- } \\
\text { 1,3- dioxolan-2-ylmethyl]-1 H-1,2,4-triazole. }\end{array}$ & $25 \% \mathrm{EC}$ & Syngenta India Itd., \\
\hline 2. & Contaf & Hexaconazole & $\begin{array}{l}\text { (RS)-2-(2,4-dichlorophenyl)-1-(1 H-1,2,4- tri- } \\
\text { azloe - 1- yl ) hexane- } 2 \text { ol }\end{array}$ & $5 \% \mathrm{EC}$ & Rallis India Ltd., Mumbai. \\
\hline 3. & Folicur & Tebuconazole & $\begin{array}{l}\text { (RS)-1-p-chlorophenyl-4,4 dimethyl- } \\
\text { 3-(1H-1,2,4 - triazol-1-ylmethyl) penton-3-ol. }\end{array}$ & $25 \% \mathrm{EC}$ & Bayer India Itd., \\
\hline 4. & Bavistin & Carbendazim & Methyl-benzimidazole 2 yl carbamate & $50 \% \mathrm{WP}$ & BASF India Ltd., Mumbai. \\
\hline 5. & Cabriotop & $\begin{array}{l}\text { Pyraclostrobin } 5 \% \\
+ \text { Metiram } 55 \%\end{array}$ & - & $60 \%$ WG & BASF India Ltd., Mumbai. \\
\hline 6. & Headline & Pyraclostrobin & $\begin{array}{l}\text { Carbamic acid,[2-[[[ 1- (4- chlorophenyl)-1 H } \\
\text {-pyrazole-3-yl]oxy]methyl]methoxy- methyl } \\
\text { ester] }\end{array}$ & $20 \%$ WG & BASF India Ltd., Mumbai. \\
\hline 7. & Polyram & Metiram & $\begin{array}{l}\text { Tris[ammine[ethylenebis(dithiocarbamato)] } \\
\text { zinc( } 2+) \text { ] [tetrahydro-1,2,4,7-dithiadiazo- } \\
\text { cine-3,8-dithione],polymer }\end{array}$ & $70 \%$ WG & BASF India Ltd., Mumbai. \\
\hline 8. & $\begin{array}{l}\text { Dithane M- } \\
45\end{array}$ & Mancozeb & $\begin{array}{l}\text { [[1,2-ethanediylbis[carbamodithioa } \\
\text { to }](2-)] \text { manganese mixture with[[1,2- } \\
\text { ethanediylbis[carbamodithioato]](2-)]-zinc }\end{array}$ & $75 \%$ WP & Bayer India Itd., \\
\hline 9. & Thiram & Thiram & $\begin{array}{l}\text { Tetramethylthiuram disulfide } \\
\text { bis(dimethylthiocarbamoyl) disulfide }\end{array}$ & $75 \%$ WP & $\begin{array}{l}\text { National Pesticides and } \\
\text { chemicals, Amaravati, Ma- } \\
\text { harashtra }\end{array}$ \\
\hline 10. & Sheathmar & Validamycin & $\begin{array}{l}\text { 1,5,6-Trideoxy-3-O-B-D-Glucopyranosyl- } \\
\text { 5-hydroxymethyl-1-((4,5,6-Trihydroxy- } \\
\text { 3-Hydroxymethyl)-2-cyclohexen-1-yl)amino) } \\
\text { D-chiro-Inositol }\end{array}$ & $3 \% \mathrm{~L}$ & $\begin{array}{l}\text { Dhanuka Agritech Ltd., } \\
\text { Haryana. }\end{array}$ \\
\hline 11. & Stomp & Pendimethalin & $\begin{array}{l}\text { 3,4-dimethyl-2,6-dinitro-N-pentan-3-yl- } \\
\text { aniline }\end{array}$ & $30 \% \mathrm{EC}$ & Cynamid India Ltd. \\
\hline 12. & Atratop & Atrazine & $\begin{array}{l}\text { 2-Chloro-4-ethylamino-6-isopropylamino- } \\
\text { 1,3,5-triazine }\end{array}$ & $50 \% W P$ & Rallis India Ltd., Mumbai. \\
\hline 13. & Fernoxone & $2,4 \mathrm{D}$ & (2,4-Dichlorophenoxy) acetic acid & $80 \%$ WP & $\begin{array}{l}\text { Jai Shree Rasayan Udyog } \\
\text { Ltd., Delhi. }\end{array}$ \\
\hline 14. & Seizure & Paraquat & N,N'-dimethyl-4,4'-bipyridinium chloride, & $24 \%$ SL & $\begin{array}{l}\text { GSP crop science Pvt Ltd., } \\
\text { Ahmedabad. }\end{array}$ \\
\hline
\end{tabular}

pyraclostrobin and \% inhibition varied from 25.55 (RS11) to 87.78 (RS5) at half recommended concentration. Rice isolate was least sensitive to pyraclostrobin with $23.33 \%$ inhibition and significantly differed from all maize isolates. Whereas at recommended concentration the isolates RS1, RS4, RS6, and RS24 showed complete inhibition while least inhibition was recorded by the isolate RS16 (45.55\%) followed by RS11 (47.77\%)from Krishna and khammam districts. The rice isolate showed $47.78 \%$ inhibition and differed significantly from maize isolates except with isolate RS 11.

At half the recommended concentration of metiram, isolates
RS4, RS6, RS7, RS19, RS20 and RS21 showed cent \% inhibition in the mycelial growth, while the least inhibition was recorded by RS12 (56.66) from khammam district. Metiram has recorded $76.16 \%$ inhibition in case of rice isolate RS28 and was on par with maize isolates RS 2, RS22 and RS27. At recommended concentration per cent inhibition in the growth of isolates RS 2 (Medak), RS 4, RS 5, RS 6 (Nizamabad), RS 7, RS 8 (Karimnagar), RS 19, RS 20 and RS 21 (Guntur) was observed. Least inhibition of $72.22 \%$ was recorded by isolate RS12 (Yellandu mandal of Khammam). The rice isolate with 83.33\% inhibition significantly differed with maize isolates. 
Table 3: In vitro evaluation of fungicides against $R$. solani isolates

\begin{tabular}{|c|c|c|c|c|c|c|c|c|c|c|c|}
\hline \multirow[t]{2}{*}{ SI. No. } & \multirow{2}{*}{$\begin{array}{l}\mathrm{N} \text { a m e } \\
\text { of the } \\
\text { Isolate }\end{array}$} & \multicolumn{2}{|c|}{ Propiconazole } & \multicolumn{2}{|c|}{ Hexaconazole } & \multicolumn{2}{|c|}{ Tebuconazole } & \multicolumn{2}{|c|}{ Carbendazim } & \multicolumn{2}{|l|}{ Cabriotop } \\
\hline & & $\begin{array}{l}\mathrm{R} \\
(0.1 \%)\end{array}$ & $\begin{array}{l}\text { HR } \\
(0.05 \%)\end{array}$ & $\begin{array}{l}R \\
(0.2 \%)\end{array}$ & $\begin{array}{l}\text { HR } \\
(0.1 \%)\end{array}$ & $\begin{array}{l}R \\
(0.1 \%)\end{array}$ & $\begin{array}{l}\text { HR } \\
(0.05 \%)\end{array}$ & $\begin{array}{l}\mathrm{R} \\
0.1 \%) \\
\end{array}$ & $\begin{array}{l}\text { HR } \\
(0.05 \%)\end{array}$ & $\begin{array}{l}R \\
(0.3 \%)\end{array}$ & $\begin{array}{l}\text { HR } \\
(0.15 \%)\end{array}$ \\
\hline \multirow[t]{2}{*}{1.} & RS1 & 100.00 & 100.00 & 100.00 & 100.00 & 100.00 & 100.00 & 100.00 & 100.00 & 90.00 & 58.05 \\
\hline & & $(90.00)$ & $(90.00)$ & $(90.00)$ & $(90.00)$ & $(90.00)$ & $(90.00)$ & $(90.00)$ & $(90.00)$ & $(100.00)$ & (71.99) \\
\hline \multirow[t]{2}{*}{2.} & RS2 & 100.00 & 100.00 & 100.00 & 100.00 & 100.00 & 100.00 & 100.00 & 100.00 & 100.00 & 92.22 \\
\hline & & $(90.00)$ & $(90.00)$ & $(90.00)$ & $(90.00)$ & $(90.00)$ & $(90.00)$ & $(90.00)$ & $(90.00)$ & $(90.00)$ & $(73.82)$ \\
\hline \multirow[t]{2}{*}{3.} & RS3 & 100.00 & 100.00 & 100.00 & 100.00 & 100.00 & 100.00 & 100.00 & 100.00 & 100.00 & 100.00 \\
\hline & & $(90.00)$ & $(90.00)$ & $(90.00)$ & $(90.00)$ & $(90.00)$ & $(90.00)$ & (90.000 & (90.000 & $(90.00)$ & $(90.00)$ \\
\hline \multirow[t]{2}{*}{4.} & RS4 & 100.00 & 100.00 & 100.00 & 100.00 & 100.00 & 100.00 & 100.00 & 100.00 & 100.00 & 100.00 \\
\hline & & $(90.00)$ & $(90.00)$ & $(90.00)$ & (90.000 & $(90.00)$ & $(90.00)$ & $(90.00)$ & $(90.00)$ & $(90.00)$ & $(90.00)$ \\
\hline \multirow[t]{2}{*}{5.} & RS5 & 100.00 & 100.00 & 100.00 & 100.00 & 100.00 & 100.00 & 100.00 & 100.00 & 100.00 & 100.00 \\
\hline & & $(90.00)$ & $(90.00)$ & (90.000 & $(90.00)$ & $(90.00)$ & $(90.00)$ & $(90.00)$ & $(90.00)$ & $(90.00)$ & $(90.00)$ \\
\hline \multirow[t]{2}{*}{6.} & RS6 & 100.00 & 100.00 & 100.00 & 100.00 & 100.00 & 100.00 & 100.00 & 100.00 & 100.00 & 100.00 \\
\hline & & $(90.00)$ & $(90.00)$ & $(90.00)$ & $(90.00)$ & $(90.00)$ & $(90.00)$ & $(90.00)$ & $(90.00)$ & $(90.00)$ & $(90.00)$ \\
\hline \multirow[t]{2}{*}{7.} & RS7 & 100.00 & 100.00 & 100.00 & 100.00 & 100.00 & 100.00 & 100.00 & 100.00 & 100.00 & 100.00 \\
\hline & & $(90.00)$ & $(90.00)$ & $(90.00)$ & $(90.00)$ & $(90.00)$ & $(90.00)$ & $(90.00)$ & $(90.00)$ & $(90.00)$ & $(90.00)$ \\
\hline \multirow[t]{2}{*}{8.} & RS8 & 100.00 & 100.00 & 100.00 & 100.00 & 100.00 & 100.00 & 100.00 & 100.00 & 100.00 & 100.00 \\
\hline & & $(90.00)$ & $(90.00)$ & $(90.00)$ & $(90.00)$ & $(90.00)$ & $(90.00)$ & $(90.00)$ & $(90.00)$ & $(90.00)$ & $(90.00)$ \\
\hline \multirow[t]{2}{*}{9.} & RS9 & 100.00 & 100.00 & 100.00 & 100.00 & 100.00 & 100.00 & 100.00 & 100.00 & 100.00 & 93.33 \\
\hline & & $(90.00)$ & $(90.00)$ & $(90.00)$ & $(90.00)$ & $(90.00)$ & $(90.00)$ & $(90.00)$ & $(90.00)$ & $(90.00)$ & (75.03) \\
\hline \multirow[t]{2}{*}{10.} & RS10 & 100.00 & 100.00 & 100.00 & 100.00 & 100.00 & 100.00 & 100.00 & 100.00 & 75.03 & 69.02 \\
\hline & & $(90.00)$ & $(90.00)$ & $(90.00)$ & $(90.00)$ & $(90.00)$ & $(90.00)$ & $(90.00)$ & $(90.00)$ & (93.33) & (87.17) \\
\hline \multirow[t]{2}{*}{11.} & RS11 & 100.00 & 100.00 & 100.00 & 100.00 & 100.00 & 100.00 & 100.00 & 100.00 & 61.11 & 51.41 \\
\hline & & $(90.00)$ & $(90.00)$ & $(90.00)$ & $(90.00)$ & $(90.00)$ & $(90.00)$ & $(90.00)$ & $(90.00)$ & (76.67) & (61.11) \\
\hline \multirow[t]{2}{*}{12.} & $\mathrm{RS} 12$ & 100.00 & 100.00 & 100 & 10 & 100.00 & bo & 100.00 & 100.00 & 81.11 & 67.78 \\
\hline & & $(90.00)$ & $(90.00)$ & $(90.00)$ & $(90.00)$ & $(90.00)$ & $(90.00)$ & $(90.00)$ & $(90.00)$ & (64.24) & $(55.41)$ \\
\hline \multirow[t]{2}{*}{13.} & RS13 & 100.00 & 100.00 & 100.00 & 100.00 & 100.00 & 100.00 & 100.00 & 100.00 & 100.00 & 100.00 \\
\hline & & $(90.00)$ & $(90.00)$ & $(90.00)$ & $(90.00)$ & $(90.00)$ & $(90.00)$ & $(90.00)$ & $(90.00)$ & $(90.00)$ & $(90.00)$ \\
\hline \multirow[t]{2}{*}{14.} & RS14 & 100.00 & 100.00 & 100.00 & 100.00 & 100.00 & 100.00 & 100.00 & 100.00 & 100.00 & 100.00 \\
\hline & & $(90.00)$ & $(90.00)$ & $(90.00)$ & $(90.00)$ & $(90.00)$ & $(90.00)$ & $(90.00)$ & $(90.00)$ & $(90.00)$ & $(90.00)$ \\
\hline \multirow[t]{2}{*}{15.} & RS15 & 100.00 & 100.00 & 100.00 & 100.00 & 100.00 & 100.00 & 100.00 & 100.00 & 100.00 & 100.00 \\
\hline & & $(90.00)$ & $(90.00)$ & $(90.00)$ & $(90.00)$ & $(90.00)$ & $(90.00)$ & $(90.00)$ & $(90.00)$ & $(90.00)$ & $(90.00)$ \\
\hline \multirow[t]{2}{*}{16.} & RS16 & 100.00 & 100.00 & 100.00 & 100.00 & 100.00 & 100.00 & 100.00 & 100.00 & 90.00 & 87.78 \\
\hline & & $(90.00)$ & $(90.00)$ & $(90.00)$ & $(90.00)$ & $(90.00)$ & $(90.00)$ & $(90.00)$ & $(90.00)$ & (71.63) & (69.55) \\
\hline 17. & RS17 & 100.00 & 100.00 & 100.00 & 100.00 & 100.00 & 100.00 & 100.00 & 100.00 & 87.78 & 81.03 \\
\hline & & $(90.00)$ & $(90.00)$ & $(90.00)$ & $(90.00)$ & $(90.00)$ & $(90.00)$ & $(90.00)$ & $(90.00)$ & (69.55) & (64.18) \\
\hline 18. & RS18 & 100.00 & 100.00 & 100.00 & 100.00 & 100.00 & 100.00 & 100.00 & 100.00 & 90.00 & 85.55 \\
\hline & & $(90.00)$ & $(90.00)$ & $(90.00)$ & $(90.00)$ & $(90.00)$ & $(90.00)$ & $(90.00)$ & $(90.00)$ & (71.58) & (67.67) \\
\hline 19. & RS19 & 100.00 & 100.00 & 100.00 & 100.00 & 100.00 & 100.00 & 100.00 & 100.00 & 100.00 & 100.00 \\
\hline & & $(90.00)$ & $(90.00)$ & $(90.00)$ & $(90.00)$ & $(90.00)$ & $(90.00)$ & $(90.00)$ & $(90.00)$ & $(90.00)$ & $(90.00)$ \\
\hline
\end{tabular}




\begin{tabular}{|c|c|c|c|c|c|c|c|c|c|c|c|}
\hline \multirow[t]{2}{*}{ SI. No. } & \multirow{2}{*}{$\begin{array}{l}\mathrm{N} \text { a } \mathrm{m} \text { e } \\
\text { of the } \\
\text { Isolate }\end{array}$} & \multicolumn{2}{|c|}{ Pyraclostrobin } & \multicolumn{2}{|c|}{ Metiram } & \multicolumn{2}{|c|}{ Mancozeb } & \multicolumn{2}{|c|}{ Thiram } & \multicolumn{2}{|c|}{ Validamycin } \\
\hline & & $\begin{array}{c}R \\
(0.1 \%)\end{array}$ & $\begin{array}{c}\mathrm{HR} \\
(0.05 \%)\end{array}$ & $\begin{array}{c}R \\
(0.3 \%)\end{array}$ & $\begin{array}{c}\text { HR } \\
(0.15 \%)\end{array}$ & $\begin{array}{c}R \\
(0.25 \%)\end{array}$ & $\begin{array}{c}H R \\
(0.012 \%)\end{array}$ & $\begin{array}{c}R \\
(0.3 \%)\end{array}$ & $\begin{array}{c}H R \\
(0.015 \%)\end{array}$ & $\begin{array}{c}R \\
(0.1 \%)\end{array}$ & $\begin{array}{c}H R \\
(0.05 \%)\end{array}$ \\
\hline \multirow[t]{2}{*}{1.} & RS1 & 100.00 & 67.78 & 83.33 & 72.22 & 90.00 & 83.33 & 65.43 & 36.20 & 87.78 & 72.06 \\
\hline & & $(90.00)$ & (55.41) & (65.91) & $(58.20)$ & (71.58) & (65.93) & (53.99) & (36.98) & (69.55) & $(58.10)$ \\
\hline \multirow[t]{2}{*}{2.} & RS2 & 72.22 & 63.33 & 100.00 & 76.66 & 87.78 & 78.89 & 52.70 & 25.96 & 85.55 & 78.03 \\
\hline & & (58.220 & $(52.73)$ & $(90.00)$ & (61.13) & (69.59) & (62.69) & (26.54) & (30.63) & (67.67) & $(62.05)$ \\
\hline \multirow[t]{2}{*}{3.} & RS3 & 78.89 & 70.00 & 72.22 & 67.77 & 91.48 & 85.86 & 68.16 & 33.20 & 88.33 & 80.00 \\
\hline & & $(62.66)$ & (56.79) & $(58.22)$ & (55.43) & (73.05) & $(67.92)$ & $(55.65)$ & (35.18) & (70.07) & (63.44) \\
\hline \multirow[t]{2}{*}{4.} & RS4 & 100.00 & 83.33 & 100.00 & 100.00 & 100.00 & 100.00 & 73.63 & 33.66 & 100.00 & 92.22 \\
\hline & & $(90.00)$ & $(66.02)$ & $(90.00)$ & $(90.00)$ & $(90.00)$ & $(90.00)_{-}$ & (59.10) & $(35.46)$ & $(90.00)$ & $(73.82)$ \\
\hline \multirow[t]{2}{*}{5.} & RS5 & 76.66 & 87.78 & 100.00 & 92.22 & 100.00 & 100.00 & 56.73 & 27.90 & 93.33 & 85.50 \\
\hline & & $(61.15)$ & $(69.55)$ & $(90.00)$ & $(73.81)$ & $(90.00)$ & $(90.00)$ & $(48.87)$ & $(31.88)$ & (75.04) & $(67.62)$ \\
\hline \multirow[t]{2}{*}{6.} & RS6 & 100.00 & 83.33 & 100.00 & 100.00 & 100.00 & 100.00 & 54.50 & 26.26 & 93.33 & 85.43 \\
\hline & & $(90.00)$ & (65.91) & $(90.00)$ & $(90.00)$ & $(90.00)$ & $(90.00)$ & (47.58) & $(30.82)$ & (75.03) & (66.79) \\
\hline \multirow[t]{2}{*}{7.} & RS7 & 85.56 & 75.93 & 100.00 & 100.00 & 100.00 & 100.00 & 54.26 & 26.46 & 90.00 & 85.55 \\
\hline & & $(67.66)$ & $(60.62)$ & $(90.00)$ & $(90.00)$ & $(90.00)$ & $(90.00)$ & (47.44) & (30.95) & (71.56) & (67.66) \\
\hline \multirow[t]{2}{*}{8.} & RS8 & 83.33 & 76.31 & 100.00 & 90.00 & 100.00 & 100.00 & 61.70 & 28.26 & 87.77 & 76.26 \\
\hline & & $(65.90)$ & $(60.88)$ & $(90.00)$ & (71.56) & $(90.00)$ & $(90.00)$ & (51.76) & (32.11) & (69.53) & $(60.84)$ \\
\hline \multirow[t]{2}{*}{9.} & RS9 & 85.55 & 78.89 & 88.89 & 83.33 & 91.11 & 87.78 & 59.30 & 24.60 & 88.89 & 83.33 \\
\hline & & (67.67) & (62.65) & $(70.53)$ & $(65.90)$ & (72.65) & (69.53) & $(50.36)$ & (29.76) & (70.54) & $(65.90)$ \\
\hline \multirow[t]{2}{*}{10.} & RS10 & 61.11 & 56.66 & 78.88 & 65.55 & 76.66 & 61.11 & 40.60 & 19.20 & 83.33 & 78.88 \\
\hline & & (51.41) & $(48.83)$ & (62.64) & $(54.06)$ & (61.11) & (51.41) & (39.580 & (25.98) & $(65.90)$ & (62.64) \\
\hline \multirow[t]{2}{*}{11.} & RS11 & 47.77 & 25.55 & 80.96 & 61.11 & 72.22 & 65.55 & 33.60 & 16.23 & 81.11 & 72.22 \\
\hline & & $(43.72)$ & $(30.36)$ & $(64.13)$ & $(51.42)$ & (58.19) & (54.06) & $(35.42)$ & (23.75) & (64.24) & $(58.20)$ \\
\hline \multirow[t]{2}{*}{12.} & RS12 & 56.66 & 50.00 & 72.22 & 56.66 & 72.22 & 67.77 & 39.56 & 18.33 & 76.66 & 67.77 \\
\hline & & $(48.83)$ & $(45.00)$ & (58.19) & $(48.83)$ & (58.19) & (55.41) & (38.97) & (25.34) & (61.11) & $(55.42)$ \\
\hline \multirow[t]{2}{*}{13.} & RS13 & 81.11 & 71.40 & 86.29 & 75.16 & 100.00 & 100.00 & 54.46 & 26.60 & 83.33 & 76.66 \\
\hline & & $(64.24)$ & $(57.67)$ & (68.320 & $(60.12)$ & $(90.00)$ & $(90.00)$ & $(47.56)$ & (31.04) & $(65.90)$ & (61.11) \\
\hline \multirow[t]{2}{*}{14.} & RS14 & 82.59 & 77.41 & 85.55 & 72.22 & 100.00 & 100.00 & 62.46 & 28.50 & 78.88 & 74.44 \\
\hline & & $(65.75)$ & (61.98) & (67.700 & (58.19) & $(90.00)$ & $(90.00)$ & $(52.22)$ & $(32.26)$ & (62.64) & (59.63) \\
\hline \multirow[t]{2}{*}{15.} & RS15 & 76.66 & 71.92 & 87.78 & 78.31 & 100.00 & 100.00 & 73.16 & 32.63 & 83.33 & 76.66 \\
\hline & & (61.11) & $(58.00)$ & $(69.53)$ & $(62.24)$ & $(90.00)$ & $(90.00)$ & $(58.80)$ & (34.83) & $(65.90)$ & (61.11) \\
\hline \multirow[t]{2}{*}{16.} & RS16 & 45.55 & 27.77 & 86.67 & 80.77 & 88.44 & 83.33 & 42.50 & 20.40 & 87.78 & 70.00 \\
\hline & & $(42.44)$ & $(31.80)$ & (68.59) & $(64.00)$ & (70.12) & (65.97) & (40.68) & (26.84) & (69.55) & (56.79) \\
\hline 17. & RS17 & 65.55 & 58.53 & 87.78 & 80.92 & 90.44 & 87.78 & 47.53 & 21.40 & 85.55 & 74.60 \\
\hline & & (54.07) & (49.91) & $(69.53)$ & $(64.10)$ & (71.99) & (69.55) & (43.58) & $(27.55)$ & (67.66) & (59.74) \\
\hline 18. & RS18 & 61.11 & 54.44 & 83.33 & 76.67 & 87.33 & 78.89 & 49.50 & 23.43 & 87.78 & 81.11 \\
\hline & & $(55.42)$ & $(47.54)$ & (65.91) & $(61.12)$ & (69.15) & $(62.66)$ & (44.71) & $(28.94)$ & (69.55) & (64.24) \\
\hline 19. & RS19 & 78.89 & 67.78 & 100.00 & 100.00 & 100.00 & 100.00 & 64.63 & 30.46 & 92.22 & 85.20 \\
\hline & & (62.64) & (55.41) & $(90.00)$ & $(90.00)$ & $(90.00)$ & $(90.00)$ & (53.51) & (33.50) & $(73.80)$ & $(67.38)$ \\
\hline
\end{tabular}




\begin{tabular}{|c|c|c|c|c|c|c|c|c|c|c|c|}
\hline \multirow{2}{*}{$\begin{array}{l}\text { S I. } \\
\text { No. }\end{array}$} & \multirow{2}{*}{$\begin{array}{l}\text { Name of } \\
\text { the Isolate }\end{array}$} & \multicolumn{2}{|c|}{ Propiconazole } & \multicolumn{2}{|c|}{ Hexaconazole } & \multicolumn{2}{|c|}{ Tebuconazole } & \multicolumn{2}{|c|}{ Carbendazim } & \multicolumn{2}{|c|}{ Cabriotop } \\
\hline & & $\begin{array}{c}R \\
(0.1 \%)\end{array}$ & $\begin{array}{c}H R \\
(0.05 \%)\end{array}$ & $\begin{array}{c}R \\
(0.2 \%)\end{array}$ & $\begin{array}{c}\text { HR } \\
(0.1 \%)\end{array}$ & $\begin{array}{c}\mathrm{R} \\
(0.1 \%)\end{array}$ & $\begin{array}{c}H R \\
(0.05 \%)\end{array}$ & $\begin{array}{c}\mathrm{R} \\
0.1 \%)\end{array}$ & $\begin{array}{c}\mathrm{HR} \\
(0.05 \%)\end{array}$ & $\begin{array}{c}R \\
(0.3 \%)\end{array}$ & $\begin{array}{c}H R \\
(0.15 \%)\end{array}$ \\
\hline \multirow[t]{2}{*}{20.} & RS20 & 100.00 & 100.00 & 100.00 & 100.00 & 100.00 & 100.00 & 100.00 & 100.00 & 100.00 & 100.00 \\
\hline & & $(90.00)$ & $(90.00)$ & $(90.00)$ & $(90.00)$ & $(90.00)$ & $(90.00)$ & $(90.00)$ & $(90.00)$ & $(90.00)$ & $(90.00)$ \\
\hline \multirow[t]{2}{*}{21.} & RS21 & 100.00 & 100.00 & 100.00 & 100.00 & 100.00 & 100.00 & 100.00 & 100.00 & 100.00 & 100.00 \\
\hline & & $(90.00)$ & $(90.00)$ & $(90.00)$ & $(90.00)$ & $(90.00)$ & $(90.00)$ & $(90.00)$ & $(90.00)$ & $(90.00)$ & $(90.00)$ \\
\hline \multirow[t]{2}{*}{22.} & RS22 & 100.00 & 100.00 & 100.00 & 100.00 & 100.00 & 100.00 & 100.00 & 100.00 & 92.22 & 82.67 \\
\hline & & $(90.00)$ & $(90.00)$ & (90.00) & $(90.00)$ & $(90.00)$ & $(90.00)$ & $(90.00)$ & $(90.00)$ & (73.80) & $(65.41)$ \\
\hline \multirow[t]{2}{*}{23.} & RS23 & 100.00 & 100.00 & 100.00 & 100.00 & 100.00 & 100.00 & 100.00 & 100.00 & 100.00 & 85.55 \\
\hline & & $(90.00)$ & $(90.00)$ & $(90.00)$ & $(90.00)$ & $(90.00)$ & $(90.00)$ & $(90.00)$ & $(90.00)$ & $(90.00)$ & $(67.67)$ \\
\hline \multirow[t]{2}{*}{24.} & RS24 & 100.00 & 100.00 & 100.00 & 100.00 & 100.00 & 100.00 & 100.00 & 100.00 & 100.00 & 92.22 \\
\hline & & $(90.00)$ & $(90.00)$ & $(90.00)$ & $(90.00)$ & $(90.00)$ & $(90.00)$ & $(90.00)$ & $(90.00)$ & $(90.00)$ & (73.80) \\
\hline \multirow[t]{2}{*}{25.} & RS25 & 100.00 & 100.00 & 100.00 & 100.00 & 100.00 & 100.00 & 100.00 & 100.00 & 92.22 & 87.78 \\
\hline & & $(90.00)$ & $(90.00)$ & $(90.00)$ & $(90.00)$ & $(90.00)$ & $(90.00)$ & $(90.00)$ & $(90.00)$ & (73.81) & (69.53) \\
\hline \multirow[t]{2}{*}{26.} & RS26 & 100.00 & 100.00 & 100.00 & 100.00 & 100.00 & 100.00 & 100.00 & 100.00 & 92.22 & 85.55 \\
\hline & & $(90.00)$ & $(90.00)$ & $(90.00)$ & $(90.00)$ & $(90.00)$ & $(90.00)$ & $(90.00)$ & $(90.00)$ & (73.81) & $(67.66)$ \\
\hline \multirow[t]{2}{*}{27.} & RS27 & 100.00 & 100.00 & 100.00 & 100.00 & 100.00 & 100.00 & 100.00 & 100.00 & 100.00 & 100.00 \\
\hline & & $(90.00)$ & $(90.00)$ & $(90.00)$ & $(90.00)$ & $(90.00)$ & $(90.00)$ & $(90.00)$ & $(90.00)$ & $(90.00)$ & (90.00) \\
\hline \multirow[t]{2}{*}{28.} & RS28 (Rice) & 100.00 & 100.00 & 100.00 & 100.00 & 100.00 & 100.00 & 100.00 & 100.00 & 90.00 & 78.88 \\
\hline & & (90.00) & $(90.00)$ & (90.00) & $(90.00)$ & $(90.00)$ & $(90.00)$ & (90.00) & (90.00) & (71.58) & (62.64) \\
\hline
\end{tabular}

\begin{tabular}{|c|c|c|c|c|c|c|c|c|c|c|c|}
\hline \multirow{2}{*}{$\begin{array}{l}\text { S I } \\
\text { No. }\end{array}$} & \multirow{2}{*}{$\begin{array}{l}\text { Name of } \\
\text { the Isolate }\end{array}$} & \multicolumn{2}{|c|}{ Pyraclostrobin } & \multicolumn{2}{|c|}{ Metiram } & \multicolumn{2}{|c|}{ Mancozeb } & \multicolumn{2}{|c|}{ Thiram } & \multicolumn{2}{|c|}{ Validamycin } \\
\hline & & $\begin{array}{c}\mathrm{R} \\
(0.1 \%)\end{array}$ & $\begin{array}{c}H R \\
(0.05 \%)\end{array}$ & $\begin{array}{c}R \\
(0.3 \%)\end{array}$ & $\begin{array}{c}H R \\
(0.15 \%)\end{array}$ & $\begin{array}{c}R \\
(0.25 \%)\end{array}$ & $\begin{array}{c}H R \\
(0.012 \%)\end{array}$ & $\begin{array}{c}R \\
(0.3 \%)\end{array}$ & $\begin{array}{c}H R \\
(0.015 \%)\end{array}$ & $\begin{array}{c}R \\
(0.1 \%)\end{array}$ & $\begin{array}{c}H R \\
(0.05 \%)\end{array}$ \\
\hline \multirow[t]{2}{*}{20.} & RS20 & 74.44 & 61.11 & 100.00 & 100.00 & 100.00 & 100.00 & 61.80 & 26.96 & 92.22 & 80.00 \\
\hline & & (59.63) & $(51.42)$ & $(90.00)$ & $(90.00)$ & $(90.00)$ & $(90.00)$ & $(51.82)$ & $(31.28)$ & (73.81) & (63.43) \\
\hline \multirow[t]{2}{*}{21.} & RS21 & 76.67 & 70.53 & 100.00 & 100.00 & 100.00 & 85.55 & 57.36 & 22.36 & 93.33 & 82.00 \\
\hline & & (61.11) & $(57.12)$ & $(90.00)$ & $(90.00)$ & $(90.00)$ & $(67.66)$ & $(49.23)$ & $(28.22)$ & $(75.03)$ & (64.89) \\
\hline \multirow[t]{2}{*}{22.} & RS22 & 67.78 & 52.22 & 83.33 & 76.66 & 87.78 & 83.77 & 61.43 & 27.93 & 100.00 & 70.00 \\
\hline & & $(55.41)$ & $(46.27)$ & $(65.90)$ & $(61.11)$ & $(69.53)$ & $(66.24)$ & $(51.61)$ & (31.90) & $(90.00)$ & (56.78) \\
\hline \multirow[t]{2}{*}{23.} & RS23 & 70.00 & 47.77 & 85.55 & 74.44 & 90.00 & 86.66 & 72.53 & 35.23 & 87.77 & 65.55 \\
\hline & & (56.78) & $(43.72)$ & $(67.66)$ & $(59.63)$ & $(71.56)$ & $(68.58)$ & $(58.40)$ & $(36.41)$ & $(69.53)$ & (54.06) \\
\hline \multirow[t]{2}{*}{24.} & RS24 & 100.00 & 81.11 & 88.89 & 83.33 & 92.22 & 86.70 & 71.63 & 33.10 & 100.00 & 72.22 \\
\hline & & $(90.00)$ & (64.24) & (70.53) & $(65.90)$ & $(73.80)$ & $(68.61)$ & $(57.82)$ & $(35.12)$ & $(90.00)$ & $(58.20)$ \\
\hline \multirow[t]{2}{*}{25.} & RS25 & 54.44 & 45.56 & 90.00 & 78.89 & 90.00 & 72.22 & 57.50 & 26.56 & 81.11 & 67.78 \\
\hline & & (47.57) & $(42.45)$ & (71.58) & $(62.65)$ & $(71.63)$ & (58.19) & $(49.31)$ & (31.02) & (64.240 & (55.41) \\
\hline \multirow[t]{2}{*}{26.} & RS26 & 54.44 & 50.00 & 87.78 & 72.78 & 83.33 & 76.16 & 59.36 & 27.43 & 78.44 & 70.00 \\
\hline & & (47.55) & $(45.00)$ & (69.55) & $(58.56)$ & (65.97) & (61.120 & (50.39) & (31.58) & $(62.33)$ & (56.79) \\
\hline \multirow[t]{2}{*}{27.} & RS27 & 72.22 & 45.55 & 89.26 & 76.66 & 90.00 & 78.89 & 65.40 & 31.33 & 100.00 & 100.00 \\
\hline & & (58.19) & $(42.45)$ & (70.93) & $(61.12)$ & (71.580 & $(62.64)$ & (53.97) & $(34.03)$ & $(90.00)$ & $(90.00)$ \\
\hline \multirow[t]{2}{*}{28.} & RS28 (Rice) & 47.78 & 23.33 & 83.33 & 76.16 & 86.29 & 78.43 & 36.80 & 16.56 & 80.00 & 65.55 \\
\hline & & (43.72) & (28.87) & (65.900 & (60.77) & (68.32) & (62.33) & (37.34) & $(24.00)$ & (63.43) & (54.06) \\
\hline
\end{tabular}




\begin{tabular}{lcc}
\hline & \multicolumn{2}{c}{ Standard error $C D(p=0.05)$} \\
\hline$F_{1}$ & 0.10 & 0.20 \\
$F_{2}$ & 0.06 & 0.11 \\
$F_{3}$ & 0.22 & 0.44 \\
$F_{1} \times F_{2}$ & 0.14 & 0.29 \\
$F_{1} \times F_{3}$ & 0.55 & 1.08 \\
$F_{2} \times F_{3}$ & 0.31 & 0.62 \\
$F_{1} \times F_{2} \times F_{3}$ & 0.78 & 1.53 \\
\hline
\end{tabular}

With regard to non systemic fungicides, mancozeb was relatively inhibitorier than thiram. The isolates showed differential reaction to these fungicides at both the concentrations tested. Among the non systemic fungicides mancozeb was relatively more inhibitive than thiram, wherein it showed complete inhibition of mycelial growth in case of isolates RS4, RS5, RS6 (Nizamabad), RS7, RS8 (Karimnagar), RS13, RS14, RS15 (Warangal), RS19, RS20 and RS21 (Guntur districts) at both the concentrations except for RS21 at half recommended concentration indicating that these isolates were highly sensitive and differ from other isolates. The isolate RS10 from Bonakal mandal of Khammam district showed least (61.11) per cent inhibition at half the recommended concentration. However, the highly virulent isolates RS 11 and RS 12 from Khammam district had $72.22 \%$ inhibition to non-systemic fungicide mancozeb compared to rest of the isolates at recommended concentration. The rice isolate has recorded 78.43 and $86.29 \%$ inhibition (Table 3).

Most of the isolates showed lower inhibition with thiram in comparison with mancozeb. At half the recommended concentration the isolate RS11 (Chintakani mandal of Khammam) has recorded least \% inhibition of 16.23 while isolate RS1 showed highest inhibition of 36.20. The rice isolate had least \% inhibition of 16.56 and was on par with maize isolate RS11 (16.23) of Chintakani mandal, Khammam district, while at recommended concentration isolate RS4 from Armur mandal, Nizamabad district showed a maximum of $73.63 \%$ inhibition while the isolate RS11 recorded least inhibition of $33.60 \%$. Similarly, the per cent inhibition at recommended dosage was $36.80 \%$ in case of rice isolate and it differed significantly with maize isolates.

The sensitivity of R.solani isolates towards validamycin varied greatly from 65.55 to $100 \%$ at half the recommended concentration. Cent percent inhibition of radial growth of the isolate RS27 from Thatipadu mandal of Kurnool district was observed and the isolate RS23 from Jangareddygudem mandal of West Godavari district showed $65.55 \%$ inhibition. The rice isolate had $65.55 \%$ inhibition and differed significantly with remaining maize isolates except for RS12, RS23 and RS25 with $67.77,65.55$ and $67.78 \%$ respectively. At recommended concentration, the radial growth of the isolates RS4 (Nizamabad), RS22, RS24 (West Godavari) and RS27 (Kurnool) was completely inhibited and least per cent inhibition of 76.66 with respect to the isolate RS12 from Yellandu mandal of Khammam district. However, $80.00 \%$ inhibition was observed in rice isolate.

Interaction among the fungicides and antibiotic showed that all the isolates are relatively less sensitive against thiram at both the concentrations followed by validamycin and mancozeb. The data presented in (Table 3) indicate that the fungicides carbendazim, propiconazole, hexaconazole and tebuconazole are highly effective at both the concentrations tested. The new molecules, pyraclostrobin, metiram and cabriotop were relatively less effective at both concentrations tested. However the isolates showed differential reaction against these fungicides. The rice isolate RS28 did not show complete inhibition against pyraclostrobin, metiram and cabriotop, while fungicides carbendazim, propiconazole, hexaconazole and tebuconazole showed complete inhibition. Only four isolates viz., RS4 (Armur mandal of Nizamabad), RS22, RS24 (Eluru and Jeelugumilli mandals of West Godavari) and RS27 (Thatipadu mandal of Kurnool district) were completely inhibited by validamycin at the recommended dosage while the rice isolate recorded only $80.00 \%$ inhibition.

\subsubsection{Bio-assay of R. solani isolates with Herbicides}

In vitro evaluation of herbicides including three systemic (atrazine, 2, 4-D and Pendimethalin) and one contact (paraquat) revealed that the systemic herbicide, pendimethalin, completely inhibited the radial growth of all the $R$. solani isolates including rice isolate at recommended and half recommended concentrations tested except for the isolate RS12 from Yellandu mandal of Khammam district at half the recommended concentration.

The pre-emergence systemic herbicide pendimethalin showed complete inhibition in the mycelial growth of all the isolates at recommended concentration and at half the recommended concentration except with regard to RS12 isolate at half the recommended concentration with $85.55 \%$ inhibition (Table 4). Atrazine at recommended and half the recommended concentration showed complete inhibition in the mycelial growth of only one isolate i.e. RS7 from Jagityal mandal of Karimnagar district, while at half the recommended concentration RS17 from Tiruvur mandal of Krishna district showed least per cent inhibition of (11.11). The rice isolate recorded 67.77 and $18.89 \%$ inhibition at both the concentrations tested. The selective systemic herbicide, 2, 4-D at half the recommended concentration had maximum (65.55) \% inhibition of RS14 isolate from Atmakur mandal of Warangal district and minimum (10.0) per cent inhibition in case of RS12 from Yellandu mandal and while at recommended concentration, showed maximum inhibition of $72.22 \%$ in isolates RS3, RS7, RS14 and RS24 and minimum $18.44 \%$ in RS11 isolate, whereas 50.00 and $23.33 \%$ inhibition was observed in rice isolate at recommended and half recommended concentrations.

Contact herbicide, paraquat showed cent \% inhibition 


\begin{tabular}{|c|c|c|c|c|c|c|c|c|c|}
\hline \multirow[t]{2}{*}{ SI. No. } & \multirow{2}{*}{$\begin{array}{l}\text { Name of the } \\
\text { Isolate }\end{array}$} & \multicolumn{2}{|c|}{ Pendimethalin } & \multicolumn{2}{|c|}{ Atrazine } & \multicolumn{2}{|c|}{$2,4-D$} & \multicolumn{2}{|c|}{ Paraquat } \\
\hline & & $\mathrm{R}(0.5 \%)$ & HR (0.25\%) & $\mathrm{R}(0.5 \%)$ & HR (0.25\%) & $\mathrm{R}(0.2 \%)$ & HR (0.1\%) & $\mathrm{R}(0.5 \%)$ & HR (0.25\%) \\
\hline \multirow[t]{2}{*}{1.} & RS1 & 100.00 & 100.00 & 65.55 & 32.22 & 27.78 & 13.33 & 90.00 & 85.55 \\
\hline & & (90.00) & $(90.00)$ & (54.07) & (34.57) & $(31.80)$ & (16.41) & (71.58) & (67.67) \\
\hline \multirow[t]{2}{*}{2.} & RS2 & 100.00 & 100.00 & 72.22 & 36.42 & 25.55 & 10.00 & 92.22 & 85.26 \\
\hline & & $(90.00)$ & $(90.00)$ & (58.19) & $(37.30)$ & $(30.35)$ & (18.36) & $(73.82)$ & $(67.43)$ \\
\hline \multirow[t]{2}{*}{3.} & RS3 & 100.00 & 100.00 & 76.67 & 39.33 & 72.22 & 5.55 & 93.33 & 87.03 \\
\hline & & $(90.00)$ & $(90.00)$ & (61.11) & $(38.83)$ & (98.19) & (13.18) & (75.03) & (68.94) \\
\hline \multirow[t]{2}{*}{4.} & RS4 & 100.00 & 100.00 & 85.55 & 78.89 & 67.77 & 61.11 & 100.00 & 83.33 \\
\hline & & $(90.00)$ & $(90.00)$ & $(67.70)$ & $(62.66)$ & $(55.44)$ & $(51.42)$ & $(90.00)$ & (65.91) \\
\hline \multirow[t]{2}{*}{5.} & RS5 & 100.00 & 100.00 & 87.78 & 83.33 & 50.00 & 27.77 & 100.00 & 83.33 \\
\hline & & $(90.00)$ & $(90.00)$ & (69.59) & $(65.97)$ & $(45.00)$ & (31.75) & $(90.00)$ & $(65.93)$ \\
\hline \multirow[t]{2}{*}{6.} & RS6 & 100.00 & 100.00 & 83.33 & 76.67 & 61.11 & 41.11 & 100.00 & 72.22 \\
\hline & & $(90.00)$ & $(90.00)$ & $(65.93)$ & $(61.12)$ & (51.440 & (39.87) & $(90.00)$ & $(58.22)$ \\
\hline \multirow[t]{2}{*}{7.} & RS7 & 100.00 & 100.00 & 100.00 & 100.00 & 72.22 & 43.33 & 92.22 & 88.89 \\
\hline & & $(90.00)$ & (90.00) & $(90.00)$ & $(90.00)$ & (58.19) & (41.16) & $(73.80)$ & $(70.53)$ \\
\hline \multirow[t]{2}{*}{8.} & RS8 & 100.00 & 100.00 & 90.00 & 83.33 & 45.55 & 34.44 & 90.00 & 87.77 \\
\hline & & $(90.00)$ & (90.00) & (71.56) & $(65.90)$ & $(42.440$ & (35.93) & (71.56) & (69.53) \\
\hline \multirow[t]{2}{*}{9.} & RS9 & 100.00 & 100.00 & 91.11 & 74.44 & 58.88 & 47.77 & 88.88 & 81.00 \\
\hline & & $(90.00)$ & (90.00) & $(72.65)$ & (59.63) & $(50.11)$ & $(43.72)_{-}$ & $(70.52)$ & $(64.51)$ \\
\hline \multirow[t]{2}{*}{10.} & RS10 & 100.00 & 100.00 & 63.33 & 56.66 & 36.02 & 29.71 & 83.33 & 72.22 \\
\hline & & $(90.00)$ & $(90.00)$ & (52.74) & $(48.83)$ & (36.88) & (35.02) & $(65.90)$ & (58.20) \\
\hline \multirow[t]{2}{*}{11.} & RS11 & 100.00 & 100.00 & 50.00 & 18.89 & 18.44 & 18.89 & 90.00 & 81.11 \\
\hline & & $(90.00)$ & (90.00) & $(45.00)$ & $(25.75)$ & $(25.42)$ & $(25.75)$ & $(71.56)$ & $(64.24)$ \\
\hline \multirow[t]{2}{*}{12.} & RS12 & 100.00 & 100.00 & 61.11 & 23.33 & 27.77 & 10.00 & 87.78 & 78.88 \\
\hline & & $(90.00)$ & $(90.00)$ & $(51.42)$ & $(28.860$ & (31.69) & (18.27) & (69.53) & (62.640 \\
\hline \multirow[t]{2}{*}{13.} & RS13 & 100.00 & 100.00 & 85.55 & 43.33 & 76.66 & 61.11 & 93.33 & 84.06 \\
\hline & & $(90.00)$ & $(90.00)$ & $(67.66)$ & (41.160 & (61.11) & (51.41) & $(75.03)$ & (66.49) \\
\hline \multirow[t]{2}{*}{14.} & RS14 & 100.00 & 100.00 & 81.11 & 38.89 & 72.22 & 65.55 & 93.33 & 81.96 \\
\hline & & $(90.00)$ & $(90.00)$ & $(64.24)$ & (38.58) & (58.19) & (54.06) & (75.03) & (64.94) \\
\hline \multirow[t]{2}{*}{15.} & RS15 & 100.00 & 100.00 & 87.78 & 32.22 & 74.44 & 56.66 & 93.33 & 83.83 \\
\hline & & $(90.00)$ & $(90.00)$ & (69.53) & (34.58) & (59.63) & $(48.83)$ & (75.030 & $(66.50)$ \\
\hline \multirow[t]{2}{*}{16.} & RS16 & 100.00 & 100.00 & 54.44 & 15.55 & 48.28 & 22.32 & 67.78 & 61.11 \\
\hline & & $(90.00)$ & $(90.00)$ & $(47.54)$ & (23.44) & (44.01) & (24.09) & $(55.42)$ & $(51.42)$ \\
\hline \multirow[t]{2}{*}{17.} & RS17 & 100.00 & 100.00 & 45.55 & 11.11 & 32.22 & 14.44 & 54.44 & 45.55 \\
\hline & & $(90.00)$ & $(90.00)$ & $(42.44)$ & (16.040 & (34.580 & $(22.320$ & (47.54) & $(42.44)$ \\
\hline \multirow[t]{2}{*}{18.} & RS18 & 100.00 & 100.00 & 35.93 & 12.59 & 25.73 & 18.23 & 65.55 & 58.89 \\
\hline & & $(90.00)$ & $(90.00)$ & $(36.82)$ & (18.57) & $(30.48)$ & $(23.46)$ & $(54.06)$ & $(50.12)$ \\
\hline 19. & RS19 & 100.00 & 100.00 & 85.55 & 67.85 & 21.11 & 16.66 & 87.77 & 83.33 \\
\hline & & $(90.00)$ & $(90.00)$ & $(67.66)$ & $(55.46)$ & (27.34) & (24.09) & (69.53) & $(65.90)$ \\
\hline 20. & RS20 & 100.00 & 100.00 & 83.33 & 14.44 & 50.00 & 27.77 & 93.33 & 81.66 \\
\hline
\end{tabular}




\begin{tabular}{|c|c|c|c|c|c|c|c|c|c|}
\hline \multirow[t]{2}{*}{ SI. No. } & \multirow{2}{*}{$\begin{array}{l}\text { Name of the } \\
\text { Isolate }\end{array}$} & \multicolumn{2}{|c|}{ Pendimethalin } & \multicolumn{2}{|c|}{ Atrazine } & \multicolumn{2}{|c|}{ 2,4-D } & \multicolumn{2}{|c|}{ Paraquat } \\
\hline & & $\mathrm{R}(0.5 \%)$ & HR (0.25\%) & $\mathrm{R}(0.5 \%)$ & HR (0.25\%) & $\mathrm{R}(0.2 \%)$ & $\mathrm{HR}(0.1 \%)$ & $\mathrm{R}(0.5 \%)$ & HR (0.25\%) \\
\hline & & $(90.00)$ & $(90.00)$ & $(65.90)$ & (22.33) & $(45.00)$ & $(31.80)$ & $(75.03)$ & (64.69) \\
\hline \multirow[t]{2}{*}{21.} & RS21 & 100.00 & 100.00 & 87.77 & 70.00 & 61.11 & 50.00 & 87.77 & 81.11 \\
\hline & & (90.00) & $(90.00)$ & $(69.53)$ & (56.78) & $(51.42)$ & $(45.00)$ & $(69.53)$ & $(64.23)$ \\
\hline \multirow[t]{2}{*}{22.} & RS22 & 100.00 & 100.00 & 67.77 & 20.00 & 47.77 & 21.11 & 56.66 & 36.66 \\
\hline & & (90.00) & (90.00) & $(55.41)$ & (18.43)_ & $(43.72)$ & $(27.35)$ & $(48.83)$ & $(37.26)$ \\
\hline \multirow[t]{2}{*}{23.} & RS23 & 100.00 & 100.00 & 72.18 & 17.78 & 45.55 & 25.55 & 52.22 & 47.77 \\
\hline & & (90.00) & (90.00) & (58.17) & (26.17) & $(42.45)$ & (30.36) & $(46.27)$ & $(43.72)$ \\
\hline \multirow[t]{2}{*}{24.} & RS24 & 100.00 & 100.00 & 76.66 & 22.22 & 72.22 & 10.00 & 61.11 & 32.22 \\
\hline & & (90.00) & $(90.00)$ & $(61.11)$ & (20.44) & (58.19) & $(18.40)$ & $(51.42)$ & (34.58) \\
\hline \multirow[t]{2}{*}{25.} & RS25 & 100.00 & 100.00 & 81.11 & 45.55 & 27.77 & 12.22 & 90.00 & 85.55 \\
\hline & & (90.00) & $(90.00)$ & $(64.29)$ & $(42.44)$ & $(31.75)$ & (20.40) & $(71.56)$ & $(67.66)_{-}$ \\
\hline \multirow[t]{2}{*}{26.} & RS26 & 100.00 & 100.00 & 72.22 & 54.44 & 21.11 & 12.22 & 91.11 & 87.77 \\
\hline & & (90.00) & $(90.00)$ & $(58.20)$ & $(47.55)$ & $(27.30)$ & (19.88) & $(72.66)$ & (69.53) \\
\hline \multirow[t]{2}{*}{27.} & RS27 & 100.00 & 100.00 & 76.67 & 38.88 & 21.11 & 18.52 & 90.00 & 67.73 \\
\hline & & (90.00) & $(90.00)$ & $(61.12)$ & $(38.57)$ & $(27.30)$ & $(23.57)$ & $(71.56)$ & $(55.42)$ \\
\hline \multirow[t]{3}{*}{28.} & RS28 (Rice) & 100.00 & 100.00 & 67.77 & 18.89 & 50.00 & 23.33 & 83.33 & 76.66 \\
\hline & & (90.00) & $(90.00)$ & $(55.41)$ & $(25.75)$ & $(45.00)$ & $(28.87)$ & $(65.90)$ & (61.11) \\
\hline & & \multicolumn{2}{|c|}{ Standard error } & \multicolumn{2}{|c|}{$\mathrm{CD}(p=0.05)$} & & & & \\
\hline \multicolumn{2}{|l|}{$\mathrm{F}_{1}$} & \multicolumn{2}{|c|}{0.16} & \multicolumn{2}{|c|}{0.32} & & & & \\
\hline \multicolumn{2}{|l|}{$\mathrm{F}_{2}$} & \multicolumn{2}{|c|}{0.11} & \multicolumn{2}{|c|}{0.22} & & & & \\
\hline \multicolumn{2}{|l|}{$\mathrm{F}_{3}$} & \multicolumn{2}{|c|}{0.43} & \multicolumn{2}{|c|}{0.85} & & & & \\
\hline \multicolumn{2}{|l|}{$\mathrm{F}_{1} \times \mathrm{F}_{2}$} & \multicolumn{2}{|c|}{0.23} & \multicolumn{2}{|c|}{0.45} & & & & \\
\hline \multicolumn{2}{|l|}{$\mathrm{F}_{1} \times \mathrm{F}_{3}$} & \multicolumn{2}{|c|}{0.87} & \multicolumn{2}{|c|}{1.70} & & & & \\
\hline $\mathrm{F}_{2} \times \mathrm{F}_{3}$ & & & 61 & & 20 & & & & \\
\hline $\mathrm{F}_{1} \times \mathrm{F}_{2} \times \mathrm{F}$ & & & .23 & & .41 & & & & \\
\hline
\end{tabular}

in isolates RS4, RS5 and RS6 from Nizamabad district at recommended concentration and none of these isolates were completely inhibited at half recommended concentration. However, least inhibition (52.22) was observed in isolate RS23 from Jangareddygudem of West Godavari. While the rice isolate was inhibited by 83.33 and 76.66 at both the concentrations. At half the recommended concentration the contact herbicide paraquat showed maximum inhibition of $88.89 \%$ by RS7 from Jagityal mandal and a minimum of 32.22 by RS24 from Jeelugumilli mandal of West Godavari district.

The perusal of the data revealed that the systemic and contact herbicides showed varied sensitivity towards the $28 R$. solani isolates. Pendimethalin was highly effective in inhibiting the mycelial growth of the pathogen at both the concentrations tested followed by atrazine and 2, 4-D (Table 4).

Among the fungicides and herbicides screened in vitro against R.solani isolates, the systemic fungicides i.e carbendazim, propiconazole, hexaconazole, tebuconazole were highly effective. While similar reaction was observed with herbicide pendimethalin. The remaining fungicides (systemic and non systemic) and herbicides exhibited differential reaction. The reaction of rice isolate was in the same line as those of the maize isolates against different fungicides and herbicides tested.

The isolates exhibited differential responses against the chemicals tested. This may be explained due to the differences in genetic composition of $R$. solani populations collected from different maize growing districts of Telangana and Andhra Pradesh states. Intensive use of a particular fungicide with specific action leads to the development of resistance in the pathogen population. Hence, evaluation of a baseline data prior and after the introduction of a potential fungicide and under field conditions is necessary to study the probable disease control failure due to development of resistant strains to the fungicide during the crop period which would help in recommending the same fungicide to the farmers in the 
subsequent season. At present, BLSB disease management is mainly achieved through the use of systemic fungicides.

The findings of the study are in accordance with Sharma et al. (2002) who stated that the fungicides, Bavistin, Rhizolex, Thiophanate $\mathrm{M}$ have shown $100 \%$ inhibition of mycelial growth of R.solani f.sp. solani causing BLSB in maize, while validamycin with $56.3 \%$ inhibition at $30 \mathrm{ppm}$ under in vitro. In vitro screening of sixteen fungicides and one antibiotic against $R$. solani $f$.sp. sasakii, causing banded leaf and sheath blight in maize stated that among systemic fungicides screened, maximum mycelia growth inhibition was observed in Propiconazole and Carbendazim, while maximum inhibition of mycelium growth was observed in Carbendazim+ Mancozeb and Carbendazim+Iprodione among the combi-product fungicides tested. However, maximum mycelial growth inhibition was observed in Mancozeb and Validamycin among the non-systemic fungicides tested (Rajput et al., 2016). Propiconazole at $0.1 \%$ effectively controlled BLSB under in vivo Saxsena (2002). Effectiveness on the application of propiconazole, carbendazim against BLSB pathogen in maize has been reported by Saxena (2002). Among the test fungicides tested for the management of R.solani in maize, bavistin at $5 \mathrm{ppm}$ with $77.1 \%$ and Tilt and companion with cent \% mycelia growth inhibitions were found most effective against R.solani (Banita Devi and Thakur, 2016). Meena et al. (2003) reported that out of six fungicides (carbendazim, kitazin,bayleton, thiophenate methyl, thiram and captan) tested for their efficacy against R.solani causing BLSB, carbendazim and kitazin completely inhibited the R.solani mycelial growth even at $1 \mathrm{ppm}$ concentation. Seven fungicides of different groups were evaluated in vitro against $R$. solani f.sp.sasakii (kuhn) exner. Among these carbendazim (0.2\%) completely inhibited the growth of the pathogen. However carboxin, tridemorph and thiabendazole also proved to be effective significantly inhibiting the fungal growth (Sharma, 2006). Out of the six fungicides tested by Pujari et al. (1998) carbendazim $0.1 \%$ and validamycin $0.1 \%$ were highly effective in controlling BLSB on maize under in vivo condition. Seed and soil treatment with systemic fungicide carbendazim reduced the disease severity and per cent disease incidence of BLSB in maize (Divya, et al., 2013; Rajput and Harlapur, 2015). The efficacy of validamycin was also reported by (Ahuja and Payak, 1982). Jameel Akhtar et al. (2010) stated that bioassay studies with fungicide, carbendazim against Rhizoctonia solani causing BLSB showed $93.8 \%$ growth inhibition and was found most effective. Fungicides, pencycuron 250 followed by Folicur (tebuconazole) $250 \mathrm{EW}$, Contaf (hexaconazole) 5 EC, and Tilt (propiconazole) 25 EC, Bavistin (carbendazim) 50 WP and validamycin at higher concentrations were effective in inhibiting the rice sheath blight fungus R.solani (Vijay Krishna et al., 2009).

In the study we have evaluated the new molecules i.e strobilurins which are a new group of fungicides that are showing promising results against rice sheath blight disease control. The bio-fungicidal activity of strobilurins was reviewed and comparisons were drawn between its efficacy and the existing recommendations such as carbendazim, validamycin, and other triazoles. Strobilurins were very effective both in terms of disease reduction as well as in increasing grain yields (Biswas, 2006). Ichiba et al. (2000) worked on the respiratory activity of metominostrobin against sheath blight pathogen and concluded that mycelial cells of pathogens induce an alternate respiratory pathway in response to blockage of cytochrome pathway. However, the alternate pathway of the pathogen could also be suppressed by some flavonoids, suggesting that metominostrobin is to be used in conjunction with plant components especially when the fungicide is applied in a prophylactic manner.

Narayana Swamy et al. (2009) screened new fungicide molecules i.e Filia (tricyclazole+propiconazole), Nativo (trifloxystrobin+tebuconazole), Contaf (hexaconazole), Rhizocin (validamycin), Tilt (propiconazole) and stated that contaf @2.0 $\mathrm{ml} \mathrm{l}^{-1}$ followed by filia $2.5 \mathrm{ml} \mathrm{l}^{-1}$ were effective in controlling $R$. solani causing sheath blight of rice under in vivo.

The literature review on chemical evaluation of sheath blight of rice caused by R.solani revealed that from time to time and place to place different chemicals have been identified. Dithane M-45 (Das and Mishra, 1990), Triazole (Suryadi et al., 1989) and Carbendazim+Mancozeb (Prasad et al., 2006) were found effective.

Kataria et al. (1991) tested different fungicides against various isolates of several anastomosis groups and found variability in fungicides sensitivity between and within AGs. Knowledge on AGs, involved in a given Rhizoctonia blight outbreak and their sensitivities to different fungicides may help to facilitate selection of the most appropriate fungicide for management of the disease in any particular area or situation. Bavistin 50 WP (carbendazim), Contaf 5 EC (hexaconazole) at $0.1 \%$ and Rhizocin $3 \mathrm{~L}$ (validamycin) at $0.25 \%$ concentration were effective against rice sheath blight and sheath rot (Lore et al., 2007). The AGs of R.solani differ in their sensitivity to fungicides and can have different host ranges (Woodhall et al., 2007).

The non target effect of herbicides on soil borne pathogens and disease severity has been reviewed (Glaze et al., 1984 and Rodriguez and Curl, 1980). Four possible mechanisms were suggested to explain how plant disease could be affected by herbicides. They were direct effect on the growth of the pathogen, its virulence or the susceptibility of the host plant, and an indirect effect on the microorganisms antagonistic to the pathogen (Katan and Eshel, 1973). The possible effect of herbicides on the host plant were reported as indirect effects on the physical structure, biochemical defence or root exudations, and direct injury to plant. Such possible effects of herbicides on the host plant, the causal organism, and other micro organisms could influence the fungicidal efficacy against R.solani (Samy et al., 1993). The direct effect of herbicides on 
pathogens also has been demonstrated (El-Khadem et al., 1984 and Moustafa-Mahmoud et al., 1995).

Seven herbicides viz., pendimethalin, anilophos, paraquat, butachlor, isoproturon, alachlor and 2,4-D $\mathrm{Na}$ salt were screened for their effect on the radial growth of $R$. solani at four concentrations viz., 500, 100, 50 and 25 ppm wherein paraquat $500 \mathrm{ppm}$ caused almost complete inhibition $(99.55 \%)$ of mycelial growth of $R$.solani causing soybean aerial blight followed by 2,4-D (92.27\%) and pendimethalin (70.29\%) (Rai et al., 2000).

Three herbicides, namely 2,4-D, isoproturon and sulfosulfuron were screened against $R$.solani for growth inhibition in tomato, wherein the growth inhibition of 2,4-D, was $23.33 \%$ at $10 \mathrm{ppm}$ and $46.66 \%$ at $200 \mathrm{ppm}$ under in vitro (Kishore and Tripati, 2007). Wilkinson and Lucas (1969) have also reported paraquat being more fungitoxic than other herbicides to a range of organisms.

The detrimental effect of paraquat on mycelial growth and sclerotia production in laboratory studies suggested that paraquat may act to reduce Rhizoctonia foliar blight by direct antagonism of the pathogen (David Black et al., 1996). Incorporation of the herbicides 2,4-D [(2,4- dichlorophenoxy) acetic acid] and diuron into soil reduced the viability of propagules of Mucor piriformis (Michailides and Spotts, 1991). It is also possible that certain herbicides may alter morphology or physiology of host plants and influence their susceptibility to disease, as discussed by Altman and Rovira (1989). Ben Yephet et al. (1991) defined the ability of herbicides to reduce disease development as dependant on specific combination of herbicide, host, pathogen and soil microorganisms present.

\section{Conclusion}

Fungicides and herbicides tested, have adversely affected the pathogen causing BLSB irrespective of the locations where they have been collected and this can be effectively managed through the use of systemic fungicides i.e. Propiconozole, hexaconazole, tebuconazole and carbendazim; stating feasibility of sustainable agriculture with minimum usage of chemicals. Additional research should include fungicides and herbicide from other chemical classes and focus on their specific mechanism(s) of action in respect to inhibition of this fungal pathogen.

\section{Acknowledgement}

Main author is thankful to Head, Department of Plant Pathology, College of Agriculture, Rajendranagar, Hyderabad, Telengana State for providing basic facility to study the effectiveness of fungicides and herbicides against $R$. solani isolates.

\section{References}

Ahuja, S.C., Payak, M.M., 1982. Symptoms and signs of banded leaf and sheath blight of maize. Phytoparasitica
10, 41-49.

Akhtar, J., Jha, V.K., Kumar, A., Lal, H.C., 2009. Occurrence of banded leaf and sheath blight of maize in Jharkhand with reference to diversity in Rhizoctonia solani. Asian Journal of Agricultural Sciences 1(2), 32-35.

Altman, J., Campbell, C.L., 1977. Effect of herbicides on plant disease. Annual Reveiw of Phytopathology 15, 361-385.

Altman, J., Rovira, A.D., 1989. Herbicide pathogen interactions in soil born root diseases. Canadian Journal of Plant Pathology 11, 166-172.

Banita Devi., Thakur, B.R., 2016. Integrated Management of Banded Leaf and Sheath Blight of Maize Caused

by Rhizoctonia solani f. sp. sasakii. Proceedings of the National Academy of Sciences, India

Section B: Biological Sciences, 1-9.

Ben-Yephet, Y.S., Mhameed., Frank, Z.R., 1991. Effect of the herbicide ethalfluralin on net blotch disease of peanut pods. Plant Disease 75, 1123-1126.

Biswas, A., 2006. Activity of strobilurins fungicides against sheath blight disease of rice: an overview. Environment and Ecology 24S (Special 3), 518-520.

Bliss, C.A., 1934. The methods of probit. Sciences 79, 39.

Das, S.R., Mishra, B., 1990. Field evaluation of fungicides for control of sheath blight of rice. Indian Phytopathology 43, 94-96.

David Black, B., Russin, J.S., Griffin, J.L., Snow, J.P., 1996. Herbicide effects on Rhizoctonia solani in vitro and Rhizoctonia foliar blight of soybean (Glycine max). Weed Science 44(3), 711-716.

Divya V.R., Narayan, P.R., Uma, G.D., 2013. Management of maize banded leaf and sheath blight with fungicides and biocontrol agents. Annals of Biological Research 4(7), 179-184.

El-Khadem, M., El-Kazzaz, M.K., Hassan, M.A., 1984. Influence of different preemergence herbicides on cotton disease caused by Rhizoctonia solani and Fusarium oxysporum f. sp. vasinfectum. Plant Soil 79, 29-36.

Glaze, N.C., Dowler, C.C., Johnson, A.W., Sumner, D.R., 1984. Influence of weed control programs in intensive cropping systems. Weed Science 32, 762-767.

Ichiba, T., Kumano, K., Kashino, H., Nanba, K., Mizutani, A., Miki, N., 2000. Effect of metominostrobin on respiratory activity of Rhizoctonia solani and its efficacy for controlling rice sheath blight. Journal of Pesticide Science 25(4), 398-401.

Jameel, A., Kumar, V., Kumud, R.T., Hem Chandra, L., 2010. Integrated management of Banded Leaf and Sheath blight disease of maize. Plant Disease Research 25(1), 35-38.

Katan, J., Eshel, Y., 1973. Interaction between herbicides and plant pathogens. Residue Review 45, 147-177.

Kataria, H.R., Hugelshofer, U., Gisi, U., 1991. Sensitivity of Rhizoctonia species to different fungicides. Plant Pathology 40(2), 203-211. 
Kishore Chand, K., Tripathi, N.N., 2007. Screening of agrochemicals against Rhizoctonia solani under laboratory conditions. Agric Science Digest 27(4), 247-250.

Lore, J.S., Thind, T.S., Hunjan, M.S., Goel, R.K., 2007. Performance of different fungicides against multiple diseases of rice. Indian Phytopathology 60(3), 296-301.

Meena,. R.L., Rathore, R.S., Mathur, K., 2003. Evluation of fungicides and plant extracts against banded leaf and sheath blight of miaze. Indian Journal of Plant Protection 31(1), 94-97.

Moustafa-Mahmoud, S.M., Ragab, M.M., Sumner, D.R., Ragab, M.M., 1995. Effect of herbicides and microelements on rhizosphere microflora in relation to cotton seedling disease caused by Rhizoctonia solani (AG-4). Egyptian Journal of Agricultural Research 73(2), 333-345.

Narayan swamy, H., Sannaulla, S., Dinesh Kumar, M., 2009. Screening of new fungicides against rice sheath blight disease. Karnataka Journal of Agricultural Science 22(2), 448-449.

Nene, Y.L., Thapliyal, P.N., 1993. Fungicides in Plant Disease Control. Oxford and IBH Publication Pvt Ltd, New Delhi, 507.

Prasad, P.S., Naik, M.K., Nagaraju, P., 2006. Screening of genotypes, fungicides, botanical and bio-agents against Rhizoctonia solani, the incitant of sheath blight of rice. Proceedings of National Seminar on New Frontiers Plant Pathology, 139.

Pujari, K.C., Saikia, U.N., Bhattacharya, A., 1998. Management of banded leaf and sheath blight of maize with chemicals. Indian Phytopathology 51(1), 78-80.

Rai, J.P., Dubey, K.S., Asha Sinha., 2000. Effect of some herbicides on the growth of Rhizoctonia solani Kuhn in vitro. Indian Journal of Weed Science 32 (1/2), 112-113.

Rajput, L.S., Harlapur, S.I., 2015. Evaluation of fungicides and biocontrol agents for suppression of banded leaf and sheath blight of maize (Zea mays). Indian Phytopathology 68(2), 149-155.

Rajput L.S., Harlapur S.I., Venkatesh, I., Aggarwal, S.K., Choudhary, M., 2016. In -vitro study of fungicides and

an antibiotic against Rhizoctonia solani f. sp. sasakii causing banded leaf and sheath blight of maize. International Journal of Agriculture Sciences 8(54), 2846-2848.
Rodriguez-Kabana, R., Curl, E.A., 1980. Non target effect of pesticides on soil borne pathogens and disease. Annual Review of Phytopathology 18, 311-332.

Samy, M., Mahmoud, M., Sumner Donald, R., 1993. Interaction of fungicides, Herbicides, and planting Date with Seedling Disease of Cotton caused by Rhizoctonia solani AG-4. Plant Disease 77(1), 79-86.

Saxena, S.C., 2002. Bio-Intensive Integrated disease management of banded leaf and sheath blight of maize. In: Proceedings of $8^{\text {th }}$ Asian Regional Maize Workshop: New Technologies for the New Millennium, Bangkok, Thailand, 380-388.

Sharma, C.M., 2006. Studies on banded leaf and sheath blight of maize (Zea mays L.) caused by Rhizoctonia solani $\mathrm{f}$. sp. sasakii (kuhn) Exner. Ph.D. Thesis, Chandra Shekar Azad University of Agriculture and Technology, Kanpur.

Sharma, R.C., Vasal, S.K., Fernando Gonzalez, B.K., Batsa., Singh, N.N., 2002. Redressal of Banded leaf and sheath blight of maize through breeding, chemical and biocontrol agents. In: Proceedings of $8^{\text {th }}$ Asian Regional Maize Workshop: New Technologies for the New Millennium, Bangkok, Thailand, 390-397.

Subedi, S., 2015. A review on important maize diseases and their management in Nepal. Journal of Maize Research and Development 1(1), 28-52.

Suryadi, Y., Kadir, T.S., Sukumandi., 1989. Field evaluation of fungicides for control of sheath blight. International Rice Research Notes 14, 35.

Vijay Krishna Kumar, K., Reddy, M.S., Kloepper, J.W., Lawrence, K.S., Groth, D.E., Miller, M.E., 2009. Sheath blight disease of rice (Oryza sativa L.) - An overview. Bioscience, Biotechnology Research Asia 62, 465-480.

Wilkinson, V., Lucas, R.L., 1969. Effects of herbicides on the growth of soil fungi. New Phytologist 68, 709-719.

Woodhall, J.W., Lees, A.K., Edwards, S.G., Jenkinson, P., 2007. Characterization of Rhizoctonia solani from potato in Great Britain. Plant Pathology 56, 286-295.

Zidan, Z.H., Dahroug, S.M., El-Safety, N.A., Shehata, A.F., Ramses, S.S., 1998. Factors influencing the fungicidal activities of certain fungicides, herbicides and their binary mixtures against soil-borne fungi. In : Proc. Seventh Conference of Agricultural Development Research, Cairo, Egypt, 15-17 Dec. 1998, vol. I. Annual Agricultural Science (Cairo) Special Issue I, 277-295. 